\title{
Assessing the relative importance of flame regimes in Raman/Rayleigh line measurements of turbulent lifted flames
}

\author{
S. Hartl a,b,, R. Van Winklec, D. Geyer ${ }^{\mathrm{a}}$, A. Dreizler ${ }^{\mathrm{d}}$, G. Magnottie,f , C. Hasse ${ }^{\mathrm{b}}$, R. S. Barlow \\ ${ }^{a}$ Thermodynamics and Alternative Propulsion Systems, University of Applied Sciences \\ Darmstadt, Germany \\ ${ }^{b}$ FG Simulation of Reactive Thermo-Fluid Systems, TU Darmstadt \\ Darmstadt, Germany \\ ${ }^{c}$ University of the Pacific \\ Stockton, CA, USA \\ ${ }^{d}$ FG Reactive Flows and Diagnostics, Center of Smart Interfaces, TU Darmstadt \\ Darmstadt, Germany \\ ${ }^{e}$ Combustion Research Facility, Sandia National Laboratories \\ Livermore, CA, USA \\ ${ }^{f}$ Clean Combustion Research Center, King Abdullah University of Science and Technology \\ Thuwal 23955-6900, Saudi Arabia
}

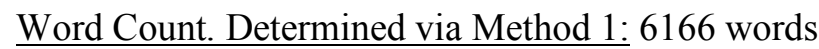

Main Text: 3645 words

Equations: $\quad($ Word count $=(\#$ equation lines + \#blank lines $) \times(7.6$ words/line $) \times(\#$ columns $))$

1. $(4+2) \times 7.6 \times 1=45.6$ words

2. $(1+2) \times 7.6 \times 1=22.8$ words

$=68.4$ words

$\underline{\text { References: }} \quad($ Word count $=(\#$ references +2$) \times(2.3$ lines $/$ reference $) \times(7.6$ words $/$ line $))$

$(27+2) \times 2.3 \times 7.6=506.92$ words

Figures: $($ Word count $=($ figure height in $\mathrm{mm}+10 \mathrm{~mm}) \times(2.2$ words $/ \mathrm{mm}) \times(\#$ columns $)+(\#$ words in caption))
1. $(93 \mathrm{~mm}+10 \mathrm{~mm}) \quad \times 2.2$ words $/ \mathrm{mm} \times 1$ column $\quad+32=258.6$ words
2. $(116 \mathrm{~mm}+10 \mathrm{~mm}) \times 2.2$ words $/ \mathrm{mm} \times 2$ columns $+73=629$ words
3. $(116 \mathrm{~mm}+10 \mathrm{~mm}) \times 2.2$ words $/ \mathrm{mm} \times 2$ columns $+93=649$ words
4. $(37 \mathrm{~mm}+10 \mathrm{~mm}) \quad \times 2.2$ words $/ \mathrm{mm} \times 1$ column $+66=169.4$ words
5. $(76.5 \mathrm{~mm}+10 \mathrm{~mm}) \times 2.2$ words $/ \mathrm{mm} \times 1$ column $\quad+50=240.3$ words

$=1946.3$ words

Preferred colloquium topic area: Turbulent flames

Color reproduction: color charges will not be paid

\footnotetext{
${ }^{*}$ Corresponding author: Mail address: Otto-Berndt-Str. 2 | 64287 Darmstadt | Germany, Phone: +49 6151 16-22899

Email address: hartl@stfs.tu-darmstadt.de (Sandra Hartl)
} 


\section{Abstract}

Understanding and quantifying the relative importance of premixed and non-premixed reaction zones within turbulent partially premixed flames is an important issue for multi-regime combustion. In the present work, the recently-developed method of gradient-free regime identification (GFRI) is applied to instantaneous 1D Raman/Rayleigh measurements of temperature and major species from two turbulent lifted methane/air flames. Local premixed and non-premixed reaction zones are identified using criteria based on the mixture fraction, the chemical explosive mode, and the heat release rate, the latter two being calculated from an approximation of the full thermochemical state of each measured sample. A chemical mode (CM) zero-crossing is a previously documented marker for a premixed reaction zone. Results from the lifted flames show strong correlations among the mixture fraction at the CM zerocrossing, the magnitude of the change in $\mathrm{CM}$ at the zero-crossing, and the local heat release rate at the $\mathrm{CM}$ zero-crossing compared to the maximum heat release rate. The trends are confirmed through a comparable analysis of numerical simulations of two laminar triple flames. These newly documented trends are associated with the transition from dominantly premixed flame structures to dominantly non-premixed flames structures. The methods introduced for assessing the relative importance of local premixed and non-premixed reactions zones have potential for application to a broad range of turbulent flames.

\section{Keywords}

GFRI, combustion regime, heat release rate, CEMA, Raman/Rayleigh 


\section{Introduction}

Partially premixed combustion is common in applications, including gas turbines and diesel engines [1-3]. The term partially premixed is used here to describe all conditions in between perfectly premixed and perfectly non-premixed. In this broad context, reactant mixtures in turbulent partially premixed flames can range from lean to rich, both inside and outside the flammability limits, such that local reaction zones can have both premixed and non-premixed characteristics. These zones contribute simultaneously, with varying dominance, to the total heat release. Consequently, knowledge of the underlying flame regimes is required for model selection [4-6].

Much of the current knowledge of fundamental structures in turbulent partially premixed flames has come from direct numerical simulation (DNS) [7-10]. In these numerical studies, several flame indices and regime identifiers have been developed for describing local reaction zones as premixed or non-premixed $[4,5,11-15]$. All of those indices and identifiers require the knowledge of 3D gradients of multiple scalars, so they are not directly applicable to experimental data. Chemical explosive mode analysis (CEMA), which relies only on the knowledge of the local thermochemical state, has also been used in analysis of DNS data to identify premixed reaction zones $[16,17]$ and extinction and ignition processes $[8,18]$. However, the analysis of experimental data in terms of the combustion regime has been conducted mostly in connection with simulations, e.g. comparing conditional averages of LES results with the experimental data $[6,19-21]$ or within an a priori/prior analysis $[6,21,22]$.

Recently, Hartl et al. [23] developed a method of regime identification based on instantaneous measurements of temperature and major species from 1D Raman/Rayleigh experiments. This socalled gradient-free regime identification (GFRI) approach is based on the concept that the experimentally accessible scalars, spatially aligned along a 1D probe volume, provide a "footprint" from which the full thermochemical state of the measured sample can be approximated. Then, from this approximated full state, the chemical mode, CM, and the heat release rate, HRR, are calculated and use in combination with the Bilger mixture fraction, $Z$, to detect and characterize premixed versus non-premixed reaction zones. These three flame markers will be defined in Section 3 . The 
GFRI method was developed and rigorously tested using fully resolved laminar flame simulations, and validated through a direct comparison of computational and experimental results at matched condition. In addition to achieving correct identification of premixed and non-premixed reaction zones in all test cases, it was shown that the heat release rates from different reaction zones can be qualitatively assessed from the measurements on a single-shot basis. From those comparisons, it was observed that: the magnitude of the change in $\mathrm{CM}$ at the zero-crossing $(\Delta \mathrm{CM})$ appeared to be positively correlated with the local HRR at the CM zero-crossing; and detected premixed reaction zones could have associated HRR far lower than the peak value associated with the non-premixed reaction zone.

In the present work, we extend the GFRI approach to include correlations among heat release rate, the mixture fraction value at the $\mathrm{CM}$ zero-crossing, and the magnitude of $\Delta \mathrm{CM}$ at the zerocrossing. This is done by analyzing experimental results from two turbulent lifted flames with different liftoff heights and numerical results from two triple flames with different stratification levels. The fully resolved triple flames are employed as counterparts of the lifted flames to validate the analysis of the experimental data. Robust trends are revealed that provide new fundamental insights on the structure of partially premixed flames by assessing the importance of flame regimes that are dominantly premixed or dominantly non-premixed.

The paper is organized as follows. Experimental and computational methods are described and the conditions of the turbulent lifted flames are given in Section 2. The recently developed gradientfree regime identification (GFRI) approach for reaction zone detection and characterization is reviewed in Section 3. Section 4 presents representative single-line experimental and numerical examples that illustrate the characteristics of predominantly premixed and predominantly nonpremixed regimes. In Section 5, robust trends correlating mixture fraction, chemical mode, and heat release rate are revealed which track the assessment of flame regimes between premixed and nonpremixed. Section 6 gives summary remarks and conclusions. 


\section{Experimental and numerical methods}

Raman/Rayleigh/LIF data of major species concentrations $\left(\mathrm{CO}_{2}, \mathrm{O}_{2}, \mathrm{CO}, \mathrm{N}_{2}, \mathrm{CH}_{4}, \mathrm{H}_{2} \mathrm{O}, \mathrm{H}_{2}\right)$ and temperature were acquired in the stabilization region of two moderately turbulent lifted flames (TLF) having jet exit Reynolds number of 2000 and 7330 (see [23] for an illustration of the lifted flame configuration). A straight tube with $8 \mathrm{~mm}$ inner diameter and $400 \mathrm{~mm}$ length was supplied with methane/air (2:1 volume ratio) and was surrounded by a laminar air co-flow of $0.3 \mathrm{~m} / \mathrm{s}$, producing average lift-off heights of $36 \mathrm{~mm}$ (TLF-36) and $130 \mathrm{~mm}$ (TLF-130) (4.5 and 16.3 jet exit diameters, respectively) based on flame luminosity. Note that the configuration TLF-130 is less stratified compared to TLF-36, due to the greater lift-off height. The laser probe volume $(6 \mathrm{~mm}$ long, $0.22 \mathrm{~mm}$ diameter, $1 / \mathrm{e}^{2}$ ) was placed within the stabilization region on one side of the annular flame, such that the leading edge of the lifted flames fluctuated above and below the probe volume position. Raman, Rayleigh, and two-photon CO-LIF signals were collected with a projected data spacing of $0.02 \mathrm{~mm}$ along the $6 \mathrm{~mm}$ probe volume. The diagnostic system, the methods of calibration and data processing, and the assessment of experimental uncertainty have been described recently in detail $[23-25]$.

The configuration investigated numerically is a 2D laminar methane/air triple flame (TF), similar to those considered in previous studies of multi-regime combustion $[4,5,23,26]$. By introducing a mixture fraction gradient $(\partial Z / \partial y)$ across the inflowing mixture, a complex flame structure is established consisting of lean and rich premixed flame branches with an embedded non-premixed flame, as illustrated in Fig. 1. The flammability limits of the methane/air mixture are $Z_{\mathrm{fl}}=[0.028$, 0.089]. Within this work, two laminar triple flames are analyzed, TF-50 and TF-200, with levels of stratification equal to $\partial Z / \partial y=50 \mathrm{~m}^{-1}$ and $\partial Z / \partial y=200 \mathrm{~m}^{-1}$, respectively. Numerical computations were performed according to Hartl et al. [23], using mixture averaged diffusion. 


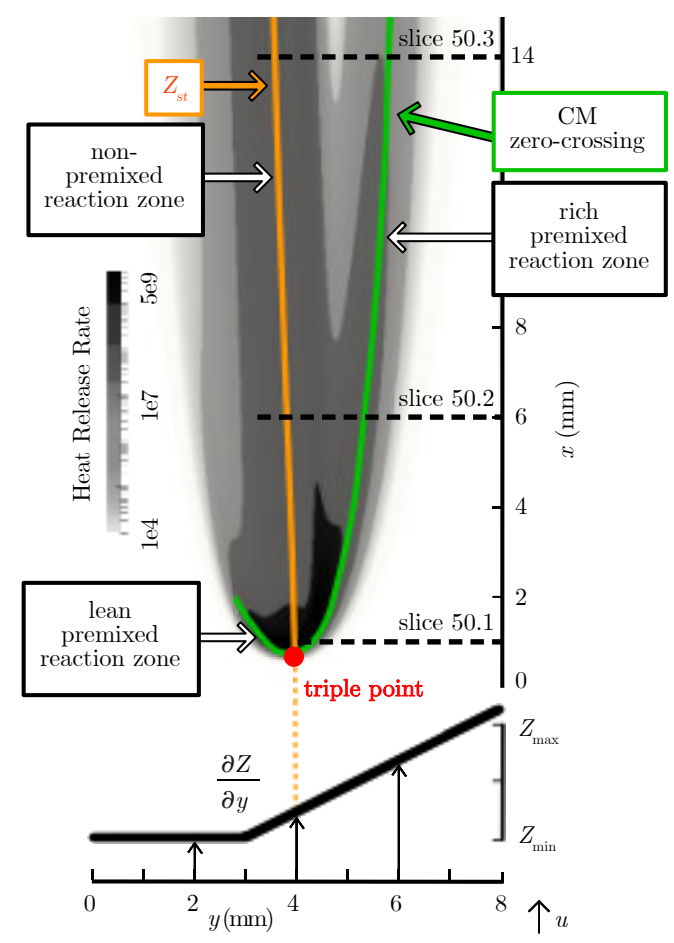

Fig. 1. Flame structure for a methane/air triple flame using a fixed level of stratification $\left(\partial Z / \partial y=50 \mathrm{~m}^{-1}\right.$, $Z \in[0,0.42])$. Positions of the slices 50.1 to 50.3 , used for the detailed analysis, are visualized by dashed lines.

\section{The gradient-free regime identification (GFRI) approach}

The GFRI approach [23] for reaction zone detection and characterization allows the local combustion regime to be identified based on Raman/Rayleigh measurements, taking into account experimental uncertainties and being independent of the spatial orientation of the experimental probe line in respect to the flame front orientation. First, a constrained homogeneous reactor calculation (constant temperature, constant pressure) is applied to approximate the full thermochemical state for each instantaneous sample, starting from just temperature and major species concentrations accessible by Raman scattering. Second, three flame markers are calculated, the Bilger mixture fraction, $Z$, the heat release rate, HRR, and the chemical mode, CM. The Bilger mixture fraction $Z$ (0-air, 1-fuel) is a normalized measure of fuel-air mixing and is calculated as:

$$
Z=\frac{\frac{2\left(Y_{\mathrm{C}}-Y_{\mathrm{C}, \mathrm{ox}}\right)}{W_{\mathrm{C}}}+\frac{Y_{\mathrm{H}}-Y_{\mathrm{H}, \mathrm{ox}}}{2 W_{\mathrm{H}}}-\frac{Y_{\mathrm{O}}-Y_{\mathrm{O}, \mathrm{ox}}}{W_{\mathrm{O}}}}{\frac{2\left(Y_{\mathrm{C}, \mathrm{fuel}}-Y_{\mathrm{C}, \mathrm{ox}}\right)}{W_{\mathrm{C}}}+\frac{Y_{\mathrm{H}, \mathrm{fuel}}-Y_{\mathrm{H}, \mathrm{ox}}}{2 W_{\mathrm{H}}}-\frac{Y_{\mathrm{O}, \mathrm{fuel}}-Y_{\mathrm{O}, \mathrm{ox}}}{W_{\mathrm{O}}}}
$$


The subscripts $\mathrm{C}, \mathrm{H}$ and $\mathrm{O}$ correspond to the elements. The symbol $W$ refers to their atomic masses, and $Y$ to their elemental mass fractions. The HRR is a local quantity which can be used to detect reaction zones and their relative importance in laminar and turbulent flames. It is calculated as:

$$
\mathrm{HRR}=-1 / \rho \sum_{i=1}^{N_{S}} \dot{\omega}_{\mathrm{i}} h_{f, i}
$$

with the chemical source term $\dot{\omega}_{\mathrm{i}}$ and $h_{f, i}$ being the enthalpy of formation of species $i$.

Chemical explosive mode analysis (CEMA) [17], has been developed for analyzing direct numerical simulations of turbulent flames. Starting from the balance equations of a chemically reacting system, the eigenvalue with the largest real part of the chemical Jacobian, $\lambda_{\mathrm{e}}$, can be used to detect reaction zones associated with spatial/temporal changes in the modes of a mixture. As in [16, 23] we use the expression $C M=\operatorname{sign}\left(\operatorname{Re}\left(\lambda_{e}\right)\right) \times \log _{10}\left(1+\left|\operatorname{Re}\left(\lambda_{e}\right)\right|\right)$, rather than $\operatorname{Re}\left(\lambda_{e}\right)$ directly, where "sign" denotes the signum function and Re the real part.

In the GFRI approach a premixed reaction zone is identified by a CM zero-crossing combined with significant HRR values at the CM zero-crossing, and a non-premixed reaction zone is identified by negative $\mathrm{CM}$ and significant $\mathrm{HRR}$ values at the stoichiometric mixture fraction, $Z_{\mathrm{st}}$. The $\mathrm{CM}$ results from the approximated thermochemical states were shown to be in good agreement with the full simulation, including both the spatial location of the CM zero-crossing and the CM values across the reaction zones [23]. Note that the GFRI approach was originally developed only to identify premixed and non-premixed reaction zones. However, the HRR results were sufficiently accurate for a qualitative assessment of the relative importance of different reaction zones within the same overall flame structure. The present work builds upon this observation and analyzes the relative importance of reaction zones by means of the HRR and the magnitude of the change in $\mathrm{CM}$ at the zero-crossing $(\Delta \mathrm{CM})$

\section{Analysis of individual sample lines}

Figure 2 shows representative profiles along the spatial coordinate $y$ of the temperature, the chemical mode, the heat release rate, and the mixture fraction derived from Raman/Rayleigh 
measurements in the two turbulent lifted flames. Reaction zones identified using the GFRI approach are indicated as vertical lines; blue for non-premixed and red for premixed. Further, the abrupt change in the chemical mode at the $\mathrm{CM}$ zero-crossing, $\triangle \mathrm{CM}$, is visualized as a marker for the strength of a premixed reaction zone. The positions of stoichiometric mixture fraction, maximum $\mathrm{HRR}$, and the $\mathrm{CM}$ zero-crossing are marked along the mixture fraction curve referred to as $Z_{\mathrm{st}}, Z_{\mathrm{HRR}}$ and $Z_{\mathrm{CM}}$, respectively. Three examples are considered for each of the turbulent lifted flames, TLF130 (left column) and TLF-36 (right column), to illustrate the range of observed flame structures. For each flame the samples are numbered in order of decreasing $\Delta \mathrm{CM}$. Note that these examples emphasize fuel-rich regions because, as discussed in [23], the rich premixed branch in the experimental lifted flame and the numerical triple flame extends farther downstream than the lean premixed branch. Therefore, realizations having a rich-premixed zone and a non-premixed zone but no lean premixed zone are most common. Corresponding examples for lean regions are omitted due to space limitations. However, the analysis in Section 5 includes lean and rich cases. 


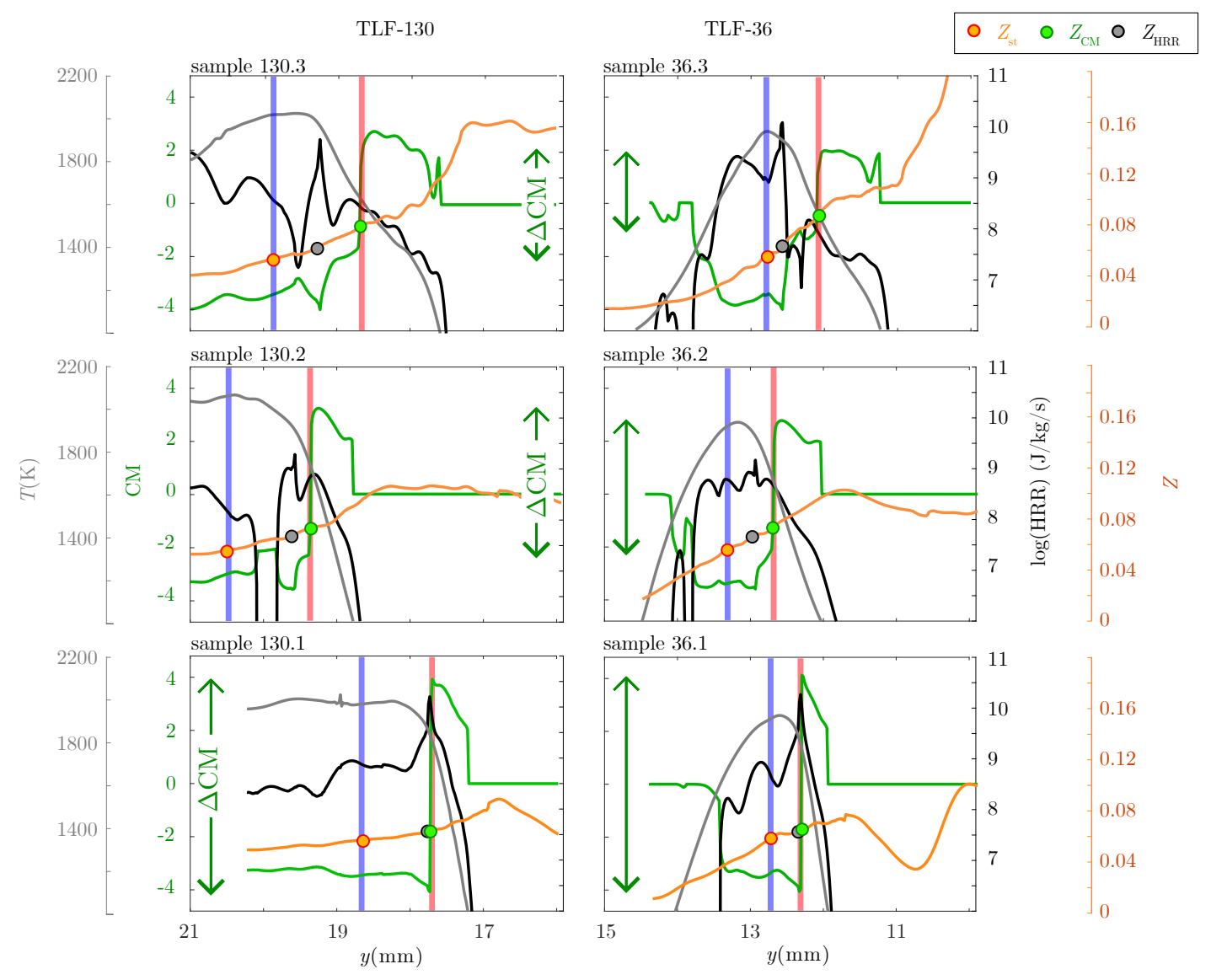

Fig. 2. Representative profiles of $T$ (grey), CM (green), HRR (black), and $Z$ (orange) from the turbulent lifted flames TLF-130 (left column) and TLF-36 (right column). Vertical lines in each subfigure denote the position of the premixed (red) and non-premixed (blue) reaction zones. The positions for stoichiometry, the $\mathrm{CM}$ zero-crossing, and $\mathrm{HRR}_{\max }$ are visualized along $Z$ as orange, green and black dots. The change in the CM at the $\mathrm{CM}$ zero-crossing, $\Delta \mathrm{CM}$, is also indicated.

Each of the six samples in Fig. 2 shows a single CM zero-crossing and a corresponding significant HRR at a fuel-rich location in mixture fraction, indicating a rich premixed reaction zone. Samples 130.1 (lower stratification) and 36.1 (higher stratification), shown in the bottom row, reveal that $\mathrm{HRR}_{\max }$ is located directly at the $\mathrm{CM}$ zero-crossing $\left(Z_{\mathrm{CM}}=Z_{\mathrm{HRR}}=0.063\right)$. These maxima are significantly larger (more than factor 10 ) than the HRR values in the negative CM region around $Z_{\text {st }}$, which corresponds to the non-premixed reaction zone. Further, there is a sharp change in CM at the zero-crossing $(\Delta \mathrm{CM} \sim 8)$, going from the overall maximum to the overall minimum in each case. Thus, the bottom frames show examples of instantaneous flame structures that are dominantly premixed and slightly rich in character; the premixed reaction zone dominates the overall heat 
release profile. For samples 130.2 and 36.2 (middle row) the CM zero-crossings is positioned at richer conditions $\left(Z_{\mathrm{CM}}=0.0735\right)$. There is a local maximum in the HRR at the $\mathrm{CM}$ zero-crossing, but $\mathrm{HRR}_{\max }$ has shifted away from the $\mathrm{CM}$ zero-crossing $\left(Z_{\mathrm{HRR}}=0.068\right)$. Furthermore, $\Delta \mathrm{CM}$ is smaller than in samples 130.1 and 36.1. More specifically, the initial sharp decrease in CM at the zerocrossing is followed by a more gradual decay towards the minimum value. That $\mathrm{CM}$ minimum occurs at the location of $H R R_{\max }$, which is near $Z=0.065$ in both samples. This is consistent with the $\mathrm{HRR}_{\max }$ location in pure non-premixed methane/air flames [23, 27]. Following, samples 130.2 and 36.2 can be described as blended structures in which non-premixed and premixed reaction zones are closely associated in physical and mixture fraction space and both contribute to the overall heat release. For samples 130.3 and 30.3 (top row) the CM zero-crossings occur at even richer conditions $\left(Z_{\mathrm{CM}}=0.083,0.089\right.$ for sample 130.3 and sample 36.3 , respectively), $\Delta \mathrm{CM}$ is further reduced, and the HRR values at the $\mathrm{CM}$ zero-crossing $\left(H R R_{C M}\right)$ are lower than $H_{R R} R_{\max }$ by more than factor 10 . Accordingly, the reaction zones in samples 130.3 and 36.3 are dominantly non-premixed in character.

In summary, a predominantly premixed flame structure is characterized by a HRR maximum at the $\mathrm{CM}$-zero-crossing and large $\Delta \mathrm{CM}$ values. A dominant non-premixed reaction flame structure is characterized by $H R R_{\max }$ occurring within the negative $\mathrm{CM}$ region slightly richer than $Z_{\text {st }}$ and by a weak $\mathrm{CM}$ zero-crossing (small $\triangle \mathrm{CM}$ ) with $\mathrm{HRR}_{\mathrm{CM}}$ values much lower than $\mathrm{HRR}_{\max }$. Thus, the relative strength of the rich premixed reaction zone, as quantified by $H R R_{C M}$ and $\Delta C M$, appears to be strongly dependent on the location of the $\mathrm{CM}$ zero-crossing in mixture fraction space. Both $\mathrm{HRR}_{\mathrm{CM}}$ and $\triangle \mathrm{CM}$ are decreasing with increasing $Z_{\mathrm{CM}}$.

These characteristics are further examined in Fig. 3 using the full information from the numerical results to determine $\mathrm{CM}, Z$ and $\mathrm{HRR}$ in slices from the computed laminar triple flames, again emphasizing fuel-rich mixtures. In the steady laminar triple flames the slices are taken at different downstream locations (see Fig. 1), so the transition in structure is clearly associated with the streamwise development of the flame. 


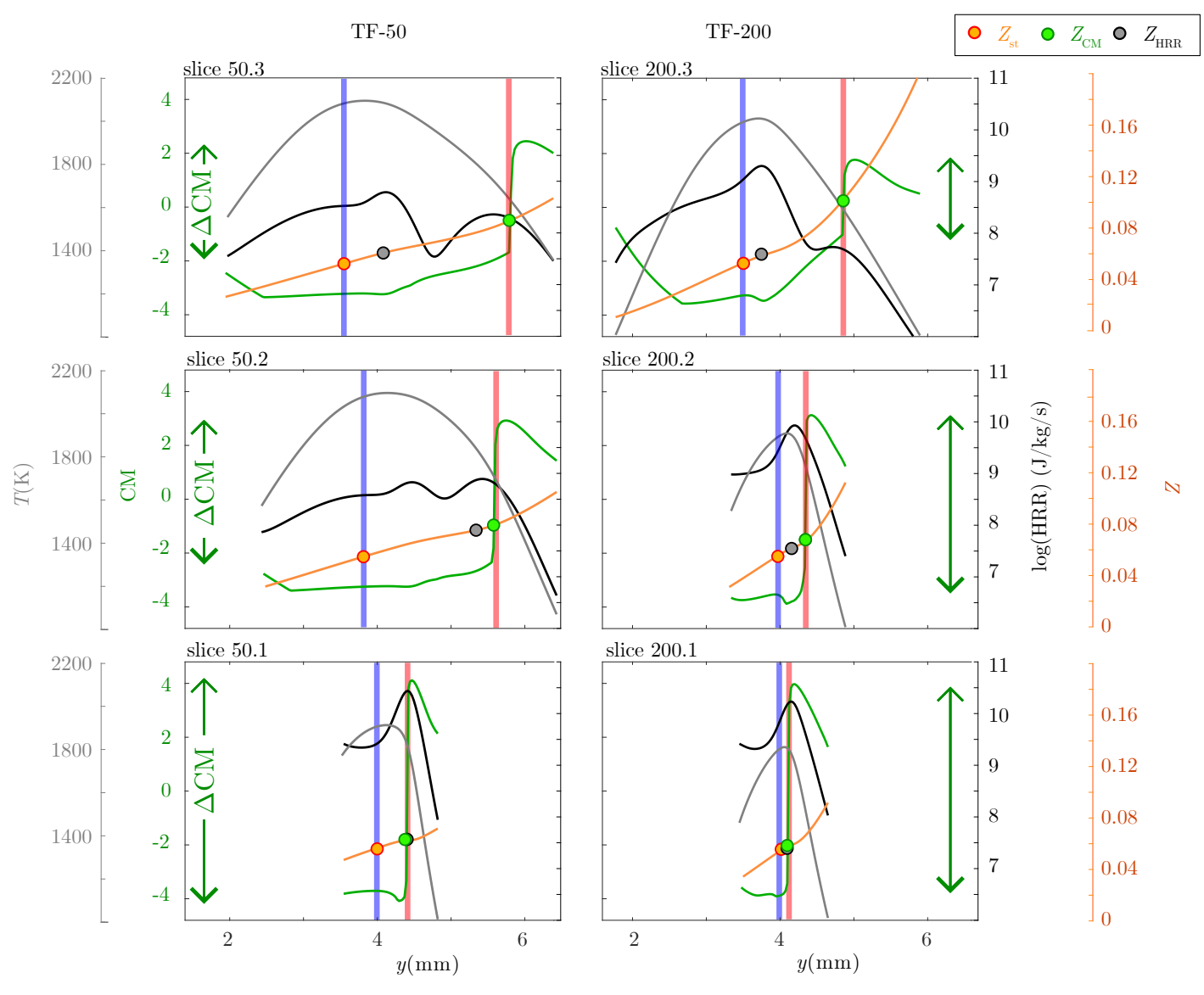

Fig. 3. Representative profiles along the radial position $y$ of $T$ (grey), CM (green), HRR (black), and $Z$ (orange) from the simulated triple flames TF-50 (left column) and TF-200 (right column). Each slice corresponds to a fixed downstream location of the triple flame, see Fig. 1. Vertical lines in each subfigure denote the position of the premixed (red) and non-premixed (blue) reaction zones, respectively. The positions for stoichiometry, the $\mathrm{CM}$ zero-crossing, and $\mathrm{HRR}_{\max }$ are visualized along $Z$ as orange, green and black dots. $\Delta \mathrm{CM}$ quantifies the changes in the chemical mode at the CM zero-crossing.

The slices close to the triple point (bottom row) each show a strong $\mathrm{CM}$ zero-crossing $(\Delta \mathrm{CM} \sim 8$ ) with $\mathrm{HRR}_{\max }$ occurring at the location of the $\mathrm{CM}$ zero-crossing, indicating a strong premixed reaction zone. In slice 200.1 (higher stratification) the premixed flame is essentially stoichiometric, and there is no discernable non-premixed zone $\left(Z_{\mathrm{CM}}=Z_{\mathrm{HRR}}=0.057\right)$. In slice 50.1 the premixed zone is shifted, due to lower stratification, to rich conditions $\left(Z_{\mathrm{CM}}=Z_{\mathrm{HRR}}=0.059\right)$, but the $\mathrm{HRR}$ at stoichiometry is an order of magnitude lower than $H_{R R}$ max. Thus, both examples are dominantly premixed. The middle row of Fig. 3 shows intermediate flame structures. In slice $50.2, H_{R} R_{\max }$ is in 
between the $\mathrm{CM}$ zero-crossing $\left(Z_{\mathrm{CM}}=0.076\right)$ and stoichiometry, but $\mathrm{HRR}_{\max }$ overlaps with the $\mathrm{CM}$ zero-crossing, suggesting that $\mathrm{HRR}_{\max }$ is mainly associated with the premixed reaction zone. However, a secondary peak that is not much lower than $H R R_{\max }$ is associated with the non-premixed reaction zone. Thus, the premixed and non-premixed zones in slice 50.2 contribute similarly to the overall heat release. In contrast, slice 200.2 has a flame structure that is spatially more compressed due to higher stratification. The $\mathrm{CM}$ zero-crossing occurs at $Z_{\mathrm{CM}}=0.068$, and $\mathrm{HRR}_{\max }$ occurs in between $Z_{\mathrm{CM}}$ and $Z_{\text {st. }}$ This can be associated with closely coupled premixed and non-premixed zones in the overall flame structure contributing to a single peak in heat release. In the slices from farthest downstream (top row) $\mathrm{HRR}_{\max }$ occurs at slightly rich conditions within the negative $\mathrm{CM}$ region and well separated from the CM zero-crossings. Thus, $H_{R} R_{\max }$ is associated with the non-premixed reaction zone, and both slices represent predominantly non-premixed flame structures. The mixture fraction values at the $\mathrm{CM}$ zero-crossing are $Z_{\mathrm{CM}}=0.082$ for slice 50.3 and $\mathrm{Z}_{\mathrm{CM}}=0.105$ for slice 200.3, while the $\triangle \mathrm{CM}$ values are roughly 4 and 3 , respectively. Note that the level of stratification has significant influence on the flame markers and on the relative HRR values in the non-premixed and premixed reaction zones. Higher stratification (higher mixture fraction dissipation) in TF-200 suppresses the strength of the rich premixed reaction zone relative to TF-50.

The experimentally investigated lifted flames and the simulated triple flames show similar trends in $\mathrm{HRR}_{\max }, \mathrm{HRR}_{\mathrm{CM}}$ and $\triangle \mathrm{CM}$ as the $\mathrm{CM}$ zero-crossing moves from near-stoichiometric toward increasingly rich conditions. Overall, the strength of the CM zero-crossing $(\triangle \mathrm{CM})$ is directly coupled to the importance of premixed reaction zones. Further, the contribution of premixed and non-premixed reaction zones can be qualitatively analysed based on $H R R_{C M}$ and $H R R_{\max }$ and their positions relative to each other.

\section{Assessing the relative importance of local reaction zones}

In this section, the analysis is extended from the trends observed in the single sample lines to include a statistical significant number of samples. More than 500 Raman/Rayleigh sample lines 
(300 data points per $6 \mathrm{~mm}$ line) were processed for the two lifted flames, where some of these samples showed more than one CM zero-crossing, hence more than one premixed reaction zone. All experimental results, including both rich and lean conditions are included. These are again compared against the triple flame simulations results from lean- and rich-side trajectories with increasing vertical distances, as depicted in Fig. 1.

First, in Fig. 4, $\Delta \mathrm{CM}$ is plotted against $Z_{\mathrm{CM}}$. The single line sample points from Fig. 2 for the two lifted flames are shown as orange dots, corresponding to predominantly premixed $(\Delta \mathrm{CM}=8.5,8.8)$, intermediate $(\Delta \mathrm{CM}=4.5,4.1)$, and the interaction of premixed with predominantly non-premixed flame regimes $(\triangle \mathrm{CM}=3,2.6)$ for TLF-130 and TLF-36, respectively.

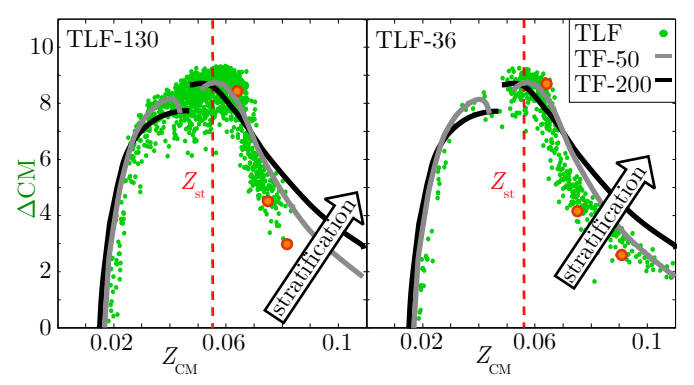

Fig. 4. $\Delta \mathrm{CM}$ plotted against $Z_{\mathrm{CM}}$ from the lifted flames TFL-130 and TFL-36 (green scatter) and from the laminar triple flames TF-50 and TF-200 (lines). The position of stoichiometry is marked as red dashed line and the trend of higher initial stratification for the triple flames is visualized by an arrow. Orange dots denote the single line sample points of the turbulent lifted flames used in Section 4.

The largest $\Delta \mathrm{CM}$ values arise near $Z_{\text {st }}$ indicating strong $\mathrm{CM}$ zero-crossings and thus dominant premixed reaction zones. The actual $\triangle \mathrm{CM}$ peak value is situated slightly on the rich side. For both rich and lean mixtures, $\triangle \mathrm{CM}$ decreases and tends toward zero (weak or no $\mathrm{CM}$ zero-crossing). However, the lean and rich sides show different trends towards zero $\Delta \mathrm{CM}$. On the lean side, $\Delta \mathrm{CM}$ drops very rapidly, and trajectories are similar for the different stratification levels. On the rich side, a fixed $\triangle \mathrm{CM}$ value extends to a higher mixture fraction in flames with higher stratification (TLF-36 and TF-200). The turbulent lifted flames and the laminar triple flames show similar characteristics in $\Delta \mathrm{CM}$, with good qualitative agreement and a clear progression from strong premixed (high $\Delta \mathrm{CM}$ ) to weak premixed reaction zones (smaller $\Delta \mathrm{CM}$ ) as $Z_{\mathrm{CM}}$ moves away from $Z_{\mathrm{st}}$. 
Figure 5 presents $H R R_{C M}$ (green scatter) and the corresponding HRR $\max$ (black scatter) for all analyzed samples lines of the turbulent lifted flames and all slices of the laminar triple flames. The single line sample points from Section 4 are shown as dots $\left(H R R_{C M}\right)$ and stars $\left(H R R_{\max }\right)$.

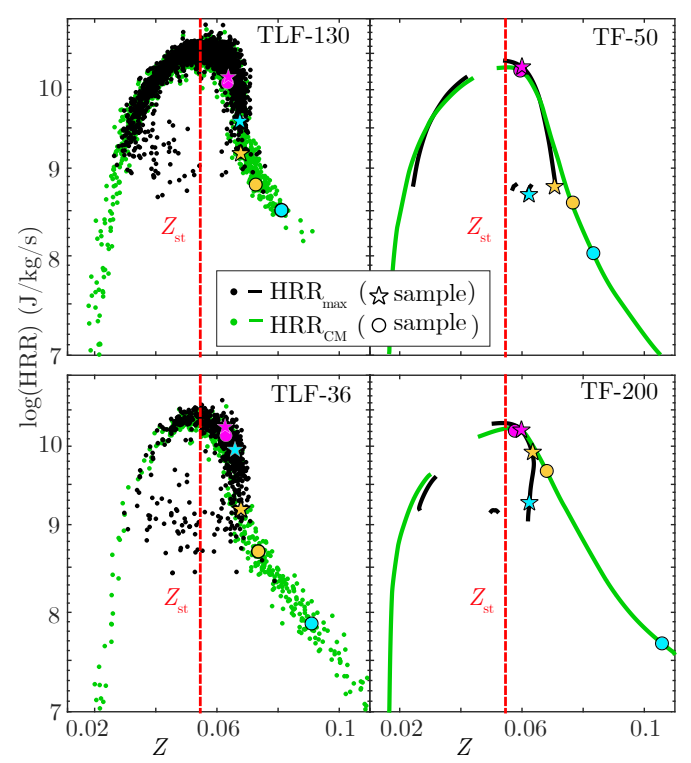

Fig. 5. Analysis of HRR $\mathrm{CM}_{\mathrm{C}}$ and $\mathrm{HRR}_{\max }$ based on TLF-130 and TLF-36 (left) and the fully resolved laminar triple flames, TF-50 and TF-200 (right). The position of stoichiometry is visualized as red dashed line. Single line sample values of $\mathrm{HRR}_{\mathrm{CM}}$ and $\mathrm{HRR}_{\max }$ from Section 4 are shown as dots and stars, respectively.

For the highest $H R R$ values near-stoichiometry, $H R R_{C M}$ and $H R R_{\max }$ occur at the same location or in very close proximity. As the CM zero-crossing moves towards richer or leaner conditions, $\mathrm{HRR}_{\mathrm{CM}}$ decreases rapidly, and the $\mathrm{HRR}_{\max }$ location separates from the $\mathrm{CM}$ zero-crossing. This separation of the heat release rates, showing the interaction of non-premixed and premixed reaction zones, is especially significant when looking at the laminar triple flame results for a fixed initial stratification. For the dominantly premixed flames (magenta stars and dots), $H R R_{\max }$ and $H R R_{\mathrm{CM}}$ are situated very close to each other. For the intermediate cases (yellow stars and dots) $H_{R} R_{\max }$ and $\mathrm{HRR}_{\mathrm{CM}}$ begin to separate. For dominantly non-premixed flames (cyan stars and dots), the difference in the mixture fraction between $H R R_{C M}$ and $H R R_{\max }$ increases. This trend also holds for the samples from the turbulent lifted flames. Again, stratification plays a significant role, shown by differences in the HRR characteristics in the laminar triple flames. 
Overall, the trends linking $Z_{\mathrm{CM}}, \Delta \mathrm{CM}, Z_{\mathrm{HRR}}$, and $\mathrm{HRR}_{\mathrm{CM}}$ and $\mathrm{HRR}_{\max }$ show a good correspondence between the turbulent lifted flames and the numerical triple flames and a transition from predominantly premixed to predominantly non-premixed flame structures as $Z_{\mathrm{CM}}$ moves toward lean or rich conditions is shown. Thus, it is possible to assess the relative importance of flame regimes based on correlations among $\mathrm{HRR}, Z_{\mathrm{CM}}$ and $\Delta \mathrm{CM}$.

\section{Summary and Conclusion}

This work has addressed the relative importance of local premixed and non-premixed reaction zones within partially premixed flames. The recently-developed gradient-free regime identification (GFRI) approach, previously shown to be sufficiently accurate for a qualitative assessment of the relative importance of different reaction zones, was applied to line-imaged measurements of temperature and major species from two turbulent lifted methane flames. In order to extend the approach with respect to the assessment of the relative importance of different reaction zones, correlations among heat release rate (HRR), chemical mode $(\mathrm{CM})$, and mixture fraction $(Z)$ were investigated. Analysis of the lifted flame results and comparisons with fully resolved laminar triple flame simulations have shown that combination of these flame markers can be used to quantitatively assess the relative importance of local premixed and non-premixed reaction zones within complex partially premixed flame structures. Predominantly premixed flames structures are characterized by a strong CM zerocrossing (high $\triangle \mathrm{CM}$ ) with a maximum $\mathrm{HRR}$ at the location of the $\mathrm{CM}$ zero-crossing. As the $\mathrm{CM}$ zero-crossing moves away from the location of maximum HRR, the premixed reaction zone progressively weakens ( $\triangle \mathrm{CM}$ and $\mathrm{HRR}_{\mathrm{CM}}$ both decrease). This trend is consistent across three orders of magnitude in the heat release rate shown from the experimental data for both lean and rich conditions. Predominantly non-premixed flame structures are characterized by a maximum in HRR close to stoichiometry (typically at slightly rich conditions) within a region of negative CM values. While for dominantly non-premixed flames one or more CM zero-crossings were still observed, the HRR at the CM zero-crossings could be orders of magnitude lower than the maximum HRR. 
Between these dominantly premixed and dominantly non-premixed cases exists a transitional range of intermediate structures in which both premixed and non-premixed reaction zones are important. This insight allows for a more detailed understanding of experimental and computational results from partially premixed flames and the relative importance of local reaction zones. The capability to assess the relative importance of premixed and non-premixed reaction zones from single-shot 1D measurements of temperature and major species in turbulent partially premixed flames gives a significant advance.

\section{Acknowledgments}

S. Hartl gratefully acknowledges funding received through the equality concept scholarship of the University of Applied Science Darmstadt. R. van Winkle is very grateful for the support by the U.S. Department of Energy, Office of Science, Office of Workforce Development for Teachers and Scientists (WDTS) under the Science Undergraduate Laboratory Internship (SULI) program. A. Dreizler acknowledges the generous support through the Gottfried Wilhelm Leibniz-program of DFG (DR 374/15-1). A. Dreizler, D. Geyer and C. Hasse acknowledge support by the German Research Foundation in the collaborative project "Multi Regime combustion under technically relevant conditions" (grant numbers DR 374/18-1, GE 2523/3-1, HA 4367/5-1). R. Barlow acknowledges support from the U.S. Department of Energy, Office of Basic Energy Sciences, Division of Chemical Sciences, Geosciences, and Biosciences. Sandia National Laboratories is a multi-mission laboratory managed and operated by National Technology and Engineering Solutions of Sandia, LLC., a wholly owned subsidiary of Honeywell International, Inc., for the U.S. Department of Energy's National Nuclear Security Administration under contract DE-NA-0003525.

\section{References}

[1] R.W. Bilger, S.H. Sterner, R.J. Kee, Combust. Flame, 80 (1990) 135-149.

[2] A.R. Masri, Proc. Combust. Inst., 35 (2015) 1115-1136.

[3] A.N. Lipatnikov, Prog. Energy Combust. Sci., 62 (2017) 87-132.

[4] E. Knudsen, H. Pitsch, Combust. Flame, 159 (2012) 242-264.

[5] E. Knudsen, H. Pitsch, Combust. Flame, 156 (2009) 678-696.

[6] M. Ihme, Y.C. See, Combust. Flame, 157 (2010) 1850-1862. 
[7] H. Wang, E.R. Hawkes, J.H. Chen, Combust. Flame, 180 (2017) 110-123.

[8] S. Lyra, B. Wilde, H. Kolla, J.M. Seitzman, T.C. Lieuwen, J.H. Chen, Combust. Flame, 162 (2015) 1234-1248.

[9] S. Karami, E.R. Hawkes, M. Talei, J.H. Chen, Combust. Flame, 169 (2016) 110-128.

[10] E.R. Hawkes, J.H. Chen, Combust. Flame, 138 (2004) 242-258.

[11] H. Yamashita, M. Shimada, T. Takeno, Proc. Combust. Inst., 26 (1996) 27-34.

[12] P. Domingo, L. Vervisch, J. Réveillon, Combust. Flame, 140 (2005) 172-195.

[13] P. Domingo, L. Vervisch, K. Bray, Combust. Theor. Model., 6 (2002) 529-551.

[14] F. Proch, A.M. Kempf, Combust. Flame, 161 (2014) 2627-2646.

[15] B. Fiorina, O. Gicquel, L. Vervisch, S. Carpentier, N. Darabiha, Combust. Flame, 140 (2005) 147-160.

[16] R. Shan, C.S. Yoo, J.H. Chen, T. Lu, Combust. Flame, 159 (2012) 3119-3127.

[17] T.F. Lu, C.S. Yoo, J.H. Chen, C.K. Law, J. Fluid Mech., 652 (2010) 45-64.

[18] I.A. Dodoulas, S. Navarro-Martinez, Combust. Theor. Model., 19 (2015) 107-129.

[19] K. Kleinheinz, T. Kubis, P. Trisjono, M. Bode, H. Pitsch, Proc. Combust. Inst., 36 (2017) 1747-1757.

[20] H. Pitsch, H. Steiner, Physics of fluids, 12 (2000) 2541-2554.

[21] A.W. Vreman, B.A. Albrecht, J.A. van Oijen, L.P.H. de Goey, R.J.M. Bastiaans, Combust. Flame, 153 (2008) $394-$ 416.

[22] Y.C. See, M. Ihme, Proc. Combust. Inst., 35 (2015) 1225-1234.

[23] S. Hartl, D. Geyer, A. Dreizler, G. Magnotti, R.S. Barlow, C. Hasse, Combust. Flame, 189 (2018) 126-141.

[24] R.S. Barlow, S. Meares, G. Magnotti, H. Cutcher, A.R. Masri, Combust. Flame, 162 (2015) 3516-3540.

[25] G. Magnotti, R.S. Barlow, Combust. Flame, 162 (2015) 100-114.

[26] H. Wu, Y.C. See, Q. Wang, M. Ihme, Combust. Flame, 162 (2015) 4208-4230.

[27] R.S. Barlow, G. Magnotti, H.C. Cutcher, A.R. Masri, Combust. Flame, 179 (2017) 117-129. 


\section{List of figure captions}

Fig. 1. Flame structure for a methane/air triple flame using a fixed level of stratification $\left(\partial Z / \partial y=50 \mathrm{~m}^{-1}, Z \in[0,0.42]\right)$. Positions of the slices 50.1 to 50.3 , used for the detailed analysis, are visualized by dashed lines.

Fig. 2. Representative profiles of $T$ (grey), CM (green), HRR (black), and $Z$ (orange) from the turbulent lifted flames TLF-130 (left column) and TLF-36 (right column). Vertical lines in each subfigure denote the position of the premixed (red) and non-premixed (blue) reaction zones. The positions for stoichiometry, the CM zero-crossing, and HRR $\max$ are visualized along $\mathrm{Z}$ as orange, green and black dots. The change in the $\mathrm{CM}$ at the $\mathrm{CM}$ zero-crossing, $\Delta \mathrm{CM}$, is also indicated.

Fig. 3. Representative profiles along the radial position $y$ of $T$ (grey), CM (green), HRR (black), and $Z$ (orange) from the simulated triple flames TF-50 (left column) and TF-200 (right column). Each slice corresponds to a fixed downstream location of the triple flame, see Fig. 1. Vertical lines in each subfigure denote the position of the premixed (red) and nonpremixed (blue) reaction zones, respectively. The positions for stoichiometry, the CM zero-crossing, and HRR max are visualized along $Z$ as orange, green and black dots. $\triangle \mathrm{CM}$ quantifies the changes in the chemical mode at the CM zerocrossing.

Fig. 4. $\triangle \mathrm{CM}$ plotted against $Z_{\mathrm{CM}}$ from the lifted flames TFL-130 and TFL-36 (green scatter) and from the laminar triple flames TF-50 and TF-200 (lines). The position of stoichiometry is marked as red dashed line and the trend of higher initial stratification for the triple flames is visualized by an arrow. Orange dots denote the single line sample points of the turbulent lifted flames used in Section 4.

Fig. 5. Analysis of HRR $\mathrm{HM}_{\mathrm{m}}$ and $H \mathrm{HR}_{\max }$ based on TLF-130 and TLF-36 (left) and the fully resolved laminar triple flames, TF-50 and TF-200 (right). The position of stoichiometry is visualized as red dashed line. Single line sample values of $\mathrm{HRR}_{\mathrm{CM}}$ and $\mathrm{HRR}_{\max }$ from Section 4 are shown as dots and stars, respectively. 